\title{
Millennium Developmental Goal and Child Survival: Bangladesh perspective.
}

Tamanna Begum

\section{Background:}

In September 2000, meeting at the United Nation (UN) Millennium summit, the world's leaders agreed to remarkable document, the Millennium Declaration ${ }^{1,2,3}$. The Millennium Developmental Goals (MDGs) agenda were adopted by the world leaders reaffirmed their share duty to "All the world's people, especially the most vulnerable and in particular, the children of the world, to whom the future belongs." They subsequently pledged to set a specific goals, the MDGs, that focus their collective efforts in the coming year as they undertake the challenges of poverty reduction and sustainable development ${ }^{1,4}$

\section{MDG Targets and Indicators ${ }^{1,2,5}$}

Goal 1: Eradicate extreme poverty and hunger.

Target 1: Reduce by half proportion of people living on less than a dollar a day.

Target 2: Reduce by half proportion of people who suffer from hunger.

Goal 2: Achieve universal primary education.

Target 3: Ensure that all boys and girls complete a full course of primary schooling.

Goal 3: Promote gender equality and empower women.

Target 4: Eliminate gender disparity in primary and secondary education preferably by 2005 at all level by 2015.

Goal 4: Reduce child mortality.

Target 5: Reduce by two-third the mortality rate among children under five.

Goal 5: Improve maternal health.

Target 6: Reduce by two three quarters the maternal mortality rate.

Goal 6: Combat HIV/AIDS, malaria and other diseases.

Target 7: Halt and begin to reverse the spread of HIV/AIDS.

Target 8: Halt and begin to reverse the incidence of malaria and other major diseases.

Goal 7: Ensure environmental sustainability.

Target 9: Integrate the principles of sustainable development into country policies and programmes, reverse loss of environmental resources.

Target 10: Reduce by half the proportion of the people without sustainable access to safe drinking water.

Target 11: Achieve significant improvement in lives of at least 100 million slum dwellers by 2020 .

Goal 8: Develop global partnership for development.

system includes a commitment to good governance,

Associate Prof. Dept. of Paediatrics ShSMC

Correspondence:

Dr.Tamanna Begum

Associate Prof. Dept. of Paediatrics ShSMC
Target 12: Develop further an open role based predictable, non- discriminatory trading and financial development and poverty reduction both nationally and internally.

Current child health status globally and as well as in Bangladesh

Globally about 11 million children under 5 years of age die annually of common preventable diseases such as acute respiratory infection, diarrhea, malaria, measles and malnutrition. Many of these deaths occur in countries in sub -Saharan Africa. The under five mortality rates (UFMR) , continue to be very high in the African Region. Eighty three percent (83\%) of the countries in the region have UFMR above 100 per 1000 live births. The high mortality is mainly due to common treatable and mostly preventable illness ${ }^{2,6,7,8}$

In Sub Saharan Africa alone, every year, some ${ }^{1,2}$ million children under five years of age die of acute respiratory infections (ARI), mainly pneumonia; 800,000 of diarrheal diseases, 500000 of measles, and about 600000 die of malaria. Each of these conditions is also associated with malnutrition in more than $50 \%$ of ensuing deaths ${ }^{4,5}$.

The late 1980s and early 1990s witnessed growing global concern about child health and the prospect of controlling illnesses and health problems. Despite the fact that adequate tools are available for effective control, childhood diseases are claiming the lives of millions of children worldwide ${ }^{6,9}$.

In May 1995 the World Health Assembly adopted IMCI as a cost effective strategy for reducing Under Five Childhood Morbidity and Mortality and promoting child growth, development and survival ${ }^{1,10}$.

In Bangladesh: This year 2007 about 30000 children under 5 years will die in Bangladesh of which about $75 \%$ will die under 1 year and 50\% will die in the neonatal periods. Most of the death will occur from the poorest 
families. But truth is that most of these deaths are preventable with low cost intervention which are available in the country ${ }^{1,4}$. Bangladesh has shown some success in reducing U5MR (under 5 mortality),

Millennium Developmental Goal and Child Survival: Bangladesh perspective.

IMR (Infant mortality rate) and NMR(neonatal mortality rate). It needs to be noted that NM (Neonatal mortality) contributes to $47 \%$ of U5MR and $63 \%$ of IMR. $50 \%$ of newborn die within first 24 hours and $80 \%$ die within first seven days giving the impression that maximum focus is needed during this time. The major causes of neonatal death: perinatal asphyxia, neonatal infection, preterm/LBW remain more or less the same $\mathrm{s}^{11,12,13}$.

\section{Child survival programs and its intervention in Bangladesh}

Bangladesh has a unique health and family welfare infrastructure where program for under 5 children are ongoing to achieve MDG 4 by 2015. Government Program for under 5 children are ${ }^{14,15}$.

1. EPI (Expanded Program of Immunization),

2. ARI (Acute respiratory tract infections),

3. CDD(control of diarrhoeal diseases),

4. IMCI(lntegrated Management of Childhood Illness),

5. School Health.

These five components are providing comprehensive service. Besides above others interventions exist like

1. Essential Newborn Care(ENC),

2. Breast feeding(BF),

3. Complementary feeding(CF),

4. Micromutrient Supplementation (Vit. A, Iron, Iodide),

5. National Nutrition Program (NNP),

6. Deworming,

7. Early Childhood Development (BCD), and

8. Newly emerging issue like childhood injury (Drowning).

In Bangladesh, the government commitment to achieve the MDGs and other mid and long term goal have increased the demand for a more regular and reliable system which enable the country to monitor its progress. Following programs are existing in our country which is directly involved to achieve the MDG4 ${ }^{3,16}$

Integrated Management of Childhood Illness (IMCI): A large number of childhood morbidity and mortality in the developing countries is caused by five conditions: Acute respiratory infections (mostly pneumonia), diarrhea, measles, malaria, or, malnutrition. The integrated Management of Childhood Illness (IMCI) strategy encompasses a range of interventions to prevent and manage this major childhood illness, both in health facilities and in the home. An integrated strategy is needed to address the overall health of children to addresses through an integrated strategy. Implementation of the IMCI strategy involves the following three components ${ }^{1,2,12}$. "Improvements in the case management skills of health staff through the provision, guidelines on integrated management of childhood illness and activities to promote".

Improvements in the health system required fo effective management of childhood". " Improvement in family and community practice". By improving the coordination and quality of existinc services, the IMCI strategy will increase tht effectiveness of care and reduce costs as to achieve the millennium development goal by reducing $\mathrm{U} 5 \mathrm{MF}^{3,6,11}$

ENC (Essential Newborn Care): Bangladesh ha: shown some success in reducing U5MR, IMR anc NMR. It needs to be noted that Neonatal mortality contributes to $47 \%$ of U5MR and $63 \%$ of IMR. 50\% o newborn die within first 24 hours and $80 \%$ die withir first seven days giving the impression that maximurr focus is needed during this time. The major causes o neonatal death are Perinatal asphyxia, neonata infections, Preterm/LBW remains more or less the same. This huge death toll on newborn lives need tc be reduced. This can be achieved by proven cos effective interventions, delivered through of-care approach $^{4,5,8}$.

Macronutrient and Micronutrient Deficiencies: Concept of cause and impact of macronutrien' deficiency on child health and child survival is wel known over the year, new concepts are coming up every day over the decades about the impact 01 various micronutrient deficiencies on child health Macronutrient and micronutrient deficiency acl together in adverse synergism in causing anc complicating malnutrition.

Of several essential micronutrients currently, zinc, vitamin A and iron deficiency are important, having significant relevance to MDG goal 4 and $5^{2,3,8}$. WHO/UNICEF recently recognized wide prevalence and impact of zinc deficiency as important public health problem threatening child survival in developing countries including Bangladesh. Zinc deficiency is one of the ten biggest factors contributing to burden of disease in developing countries with high mortality and zinc intervention are among those proposal to help reduce child death globally by $63 \%$ by WHO (MDG4) ${ }^{9,10}$.

Expanded program of immunization (EPI): EPI in Bangladesh has prevented an estimated 2 million children 
deaths and disabilities since its inception and preventing estimated 200,000 deaths each year. Coordinated effort of other child health interventions with immunization are required towards achieving MDG-4. Initially started to combat 6 vaccine preventable diseases (VPDs), EPI is now fighting against 7 diseases with inclusion of Hepatitis B since 2003. To combat 7 diseases, EPI uses 6 antigens. Most of the antigen used in Bangladesh are monovalent, only DPT is trivalent vaccine being used to prevent diphtheria, pertussis and tetanus. The antigens used in EPI are: BCG, DPT, OPV, Hepatitis -B, TTand Measles $^{4,5,12}$.

Early Childhood Development (ECD): UNICEF focuses on three areas of intervention for early childhood development: quality basic health, education and protection services; good care practices for children within the family and community; and early child development policies. UNICEF works with governments and other partners to achieve the Millennium developed goal (MDG4) ${ }^{1,2,17}$.

Complementary Feeding (IYCF): WHO and UNICEF recently resolved and endorsed the Global Strategy for infant and young child feeding. The main components are Infant should be breastfed within half an hour of birth, exclusively breastfed for the first six months of life, and thereafter should receive nutritionally adequate and safe complementary foods while breastfeeding continues up to two years and beyond.

In Bangladesh, the information on complementary food intake shows that $12 \%$ of breastfed children $<6$ months receive solid or, semisolid foods, $23 \%$ children consume liquids other than water, $18 \%$ consume animal milk, and $11 \%$ are given baby formula. Bangladesh unlike other countries in the region. Proportion of complementary feeding: Timely around $60 \%$, but whether appropriate is questionable. Exclusive Breast Feeding (EBF) rate up to 6 months$44 \%$ (BDHS-2004). ${ }^{14,15,16}$.

Control of diarrhoea! diseases (CDD): It is one of the successful program in Bangladesh. Death from diarrhea has been reduced significantly. Promoting behavioral change and improving service delivery by health personal are the main strategies for reducing diarrhoeal mortality in children under five years of age. The priority of the CDD program is promoting behavioral change of caretakers/mothers so that they provide oral rehydration therapy (ORT) to prevent dehydration due to diarrhea and improving case management by health worker. ${ }^{6,712}$

Millennium Development Goal (MDG) 4: MDG 4 goal targets to reduce the under 5 mortality in Bangladesh by two-thirds from 151 per 1000 live birth in 1990 to 51 in 2015. For MDG 4 reduce U5MR by $65 \%$, malnutrition $50 \%$ and eliminate gender disparity in child mortality and malnutrition. The other indicators of MDG4 are infant mortality rate per 1000 live birth and percentage of children under 12 months immunized against measles. Despite predictions that Bangladesh is on track to achieve MDG4 by 2015 when we look at the 19 tracking parameters enumerated at the countdown to 2015 child survival conference in London in December 2005 it appears there is little room for complacence $e^{1,2,3}$.

Though survival prospects have improved in every region, 10.5 million children died before their fifth birthday in 2004- mostly from preventable causes. The vast majority of these children (94 percent) lived in 60 countries. SubSaharan Africa, with only 20 percent of the world's young children, accounted for half of the total deaths, a situation that has shown only modest improvement ${ }^{4,8}$. In contrast, child survival has improved markedly in Latin America and the Caribbean, South Eastern and Eastern Asia and North Africa, where child mortality rates have declined by more than 3 per cent annually ${ }^{5,6,7}$.

\section{Conclusion:}

All the eight MDGs are either closely or, indirectly related to children and target date of 2015. In Bangladesh, the Governments commitment to achieve the MDGs and other long term goals have increased the demand for more regular and reliable system which enables the country to monitor its progress towards them. We will have time to reach the Millennium Development Goals worldwide and in most, or ever all, individual countries. Success will require sustained action across the entire decade between now and deadline. So we must start now. And we must ensure that more than double global developments assistance over the next few years. Nothing less will help to achieve the Goals.

\section{Reference:}

1. Progotir Pathey 2003: Bangladesh Bureau of Statistics and UNICEF.

2. State of the world children UNICEF.

3. Statistical Pocket Book of Bangladesh 2002, Bureau of Statistics.

4. A world fit for children Millenium Development Goals, special session for children document, on the rights of the child. July 2002, UNICEF.

5. 1st National Conference on Achieving Millennium Development Goats 4 and 5. March,2007.

6. Available from http//www.mdgbangla.org. Millennium development goals (MDG) issues. June 2006.

7. Available from http//www.un.org/millenniumgoal. UN Millennium Development Goals. September, 2005. 
8. Available from http//www.undp.org/mdg. Millennium Development Goals-MDGs. 2004.

9. National report on follow up to the world summit for children December. 2000. Ministry of Women and children Affairs.

10. United Nation Development Program, Millennium Development Goals-MDGs 2006.

11. UNICEF statistics. Integrated Management of Childhood Illness (IMCI) 2001.

12. World Health Organization Bangladesh. Family and community Health IMCL-2000.
13. Community IMCI: A strategy for accelerating child survival and development interventions, UNICEF by Vincent Orida 2001.

14. Global Strategy for Infant and Young Child Feeding Geneva, 2003.

15. Community-Based Strategies for Breastfeeding Promotion and Support in Developing Countries WHO 2003.Down load from websites

http://www.unicef.org/earlychildhood/index_action.html 